\title{
COMPUTATIONAL COMPLEXITY OF LCPS ASSOCIATED WITH POSITIVE DEFINITE SYMMETRIC MATRICES*
}

\author{
Yahya FATHI \\ The University of Michigan, Ann Arbor, MI, U.S.A.
}

Received 18 December 1978

Revised manuscript received 23 March 1979

\begin{abstract}
Murty in a recent paper has shown that the computational effort required to solve a linear complementarity problem (LCP), by either of the two well known complementary pivot methods is not bounded above by a polynomial in the size of the problem. In that paper, by constructing a class of LCPs-one of order $n$ for $n \geq 2$ - he has shown that to solve the problem of order $n$, either of the two methods goes through $2^{n}$ pivot steps before termination.

However that paper leaves it as an open question to show whether or not the same property holds if the matrix, $M$, in the LCP is positive definite and symmetric. The class of LCPs in which $M$ is positive definite and symmetric is of particular interest because of the special structure of the problems, and also because they appear in many practical applications.

In this paper, we study the computational growth of each of the two methods to solve the LCP, $(q, M)$, when $M$ is positive definite and symmetric and obtain similar results.
\end{abstract}

Key words: Linear Complementarity Problem, Complementary Cones, Complementary Pivot Methods, Computational Complexity, Exponential Growth.

\section{Background}

\subsection{Preliminaries}

Given a real $n$ by $n$ matrix $M$, and a real $n$-vector $q$, the Linear Complementarity Problem (LCP), denoted by $(q, M)$, is to find column vectors $w$ and $z$ in $\mathbf{R}^{n}$ such that

$$
\begin{aligned}
& w-M z=q, \\
& w \geq 0, \quad z \geq 0, \\
& w_{i} z_{i}=0 \quad \text { for all } i=1 \text { to } n
\end{aligned}
$$

(see $[1,3,4,7,12])$.

$\left(w_{j}, z_{j}\right)$ is called a complementary pair of variables, and their corresponding column vectors, $\left(I_{\cdot j},-M_{j}\right)$, is called a complementary pair of columns in this problem.

Throughout this paper if $D$ is any matrix, we denote its $j$ th column by $D_{. j}$ and

* This research is partially supported by Air Force Office of Scientific Research, Air Force Number AFOSR-78-3646. The United States Government is authorized to reproduce and distribute reprints for governmental purposes notwithstanding any copyright notation thereon. 
its $i$ th row by $D_{i \cdot}$ Let $A_{\cdot j} \in\left\{I_{\cdot j},-M_{\cdot j}\right\}$; then the ordered set $\left(A_{\cdot 1}, \ldots, A_{\cdot n}\right)$ is called a complementary set of vectors and $\operatorname{Pos}\left\{A_{\cdot_{1}}, \ldots, A_{\cdot_{n}}\right\}=\left\{y: y=\alpha_{1} A_{\cdot 1}+\cdots+\right.$ $\alpha_{n} A_{\cdot n} ; \alpha_{i} \geq 0$ for all $i=1$ to $\left.n\right\}$ is a complementary cone, in the class $\mathscr{C}(M)$ of complementary cones corresponding to the given matrix $M$. (Notice that the class $\mathscr{C}(M)$ consists of $2^{n}$ complementary cones).

A square matrix is called a $P$-matrix if all its principal subdeterminants are strictly positive. (See [11].)

Let $\left\{A_{._{1}}, \ldots, A_{._{n}}\right\}$ be a complementary set of column vectors and $H_{j}$ the hyperplane that is the linear hull of $\left\{A_{\cdot 1}, \ldots, A_{\cdot j-1}, A_{\cdot j+1}, \ldots, A_{\cdot n}\right\}$ in $\mathbf{R}^{n}$, for $j=1$ to $n$. It has been shown that if $M$ is a $P$-matrix, then the vectors $I_{j}$ and $-M_{\cdot j}$ are strictly on opposite sides of $H_{j}$ for all $j=1$ to $n$. This property is called the strict separation property [8]. It has also been shown that the LCP, $(q, M)$, has a unique solution corresponding to every $q \in \mathbf{R}^{n}$ if and only if $M$ is a $P$-matrix $[8,13]$.

Lemke and Howson developed a complementary pivot method for solving an LCP corresponding to the problem of computing an equilibrium strategy in a bimatrix game problem, and later on Lemke extended this into a method for solving a general LCP [5]. Here we will refer to it as complementary pivot method I.

Another complementary pivot method for solving LCPs is developed by Murty in [10] and we will refer to it as complementary pivot method II. See [1], [4] and [7] for a detailed discussion of these methods.

\subsection{Parametric LCP}

Consider the LCP, $(q(\gamma), M)$ where $q_{i}(\gamma)=b_{i}+\gamma b_{i}^{*}$ for $i=1$ to $n . b_{i}$ and $b_{i}^{*}$ are given real numbers, $\gamma$ is a real valued parameter and $M$ is a given $P$-matrix of order $n$. The problem of finding its solution as a function of $\gamma$ is known as the parametric LCP.

An algorithm to solve this problem is discussed in [9] and [2]. Here we briefly explain this algorithm.

Solve the LCP, $(q(\gamma), M)$ for a fixed value of $\gamma$, say $\gamma_{0}$, and find the range of values of $\gamma$ for which the current complementary basis remains feasible; say $\underline{\gamma} \leq y \leq \bar{\gamma}$. Let $r$ be the index of the variable which violates nonnegativity constraint if $\gamma$ exceeds $\bar{\gamma}$. Replace the present basic variable from the $r$ th complementary pair $\left\{w_{r}, z_{r}\right\}$ by its complement. The new basis would be feasible for $\gamma>\bar{\gamma}$. Apply a similar technique to find a feasible basis for $\gamma<\underline{\gamma}$ and repeat this process until the solution of the LCP for all values of $\gamma$ is found. For a detailed discussion of this algorithm see [9], or Problem 16.30 in [6, p. 515].

This algorithm is in fact following the line $b+b^{*} \gamma$ in $\mathbf{R}^{n}$ by continuously changing the value of $\gamma$. By the strict separation property it is clear that if this line leaves the complementary cone $\operatorname{Pos}\left(A_{\cdot 1}, \ldots, A_{\cdot n}\right)$ through the facet $\operatorname{Pos}\left(A_{\cdot 1}, \ldots, A_{\cdot j-1}, A_{\cdot j+1}, \ldots, A_{\cdot n}\right)$, then it enters the complementary cone 
$\operatorname{Pos}\left(A_{\cdot 1}, \ldots, A_{\cdot j-1}, D_{\cdot j}, A_{\cdot j+1}, \ldots, A_{\cdot n}\right)$ where $D_{\cdot j}$ is the complement of $A_{\cdot j}$ in the complementary pair of columns $\left\{I_{. j},-M_{. j}\right\}$. See Fig. 1. Hence this algorithm finds all complementary cones that the line $b+b^{*} \gamma$ cuts across as $\gamma$ varies from $-\infty$ to $+\infty$.

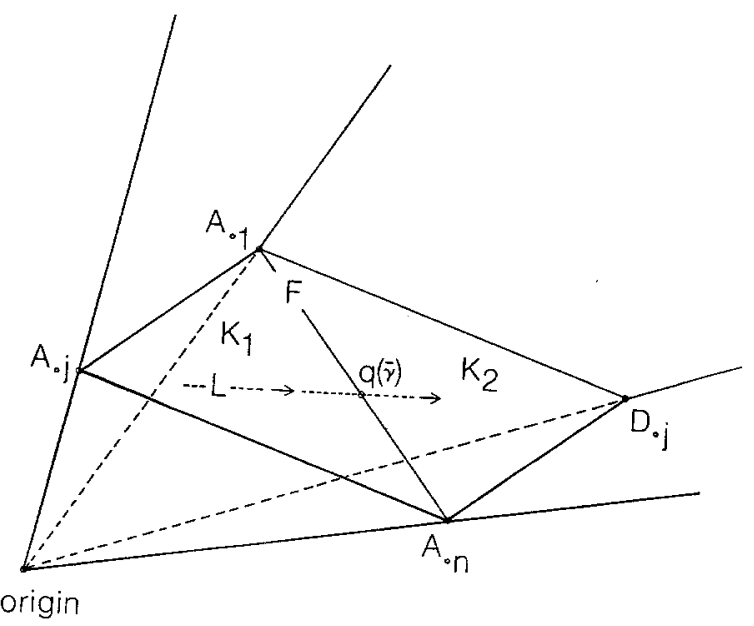

Fig. 1. Illustration of the situation in the Parametric LCP algorithm. As $\gamma$ increases through $\bar{\gamma}$, the point $q(\gamma)$ travels along the straight line $L$ in the direction of the arrow, leaves the complementary cone $K_{1}=\operatorname{Pos}\left(A_{\cdot 1}, \ldots, A_{\cdot n}\right)$ through $F=\operatorname{Pos}\left(A_{\cdot 1}, \ldots, A_{\cdot j-1}, A_{\cdot j+1}, \ldots, A_{\cdot n}\right)$ and enters the complementary cone $K_{2}=\operatorname{Pos}\left(A_{\cdot 1}, \ldots, A_{\cdot j-1}, D_{\cdot j}, A_{\cdot j+1}, \ldots, A_{\cdot n}\right)$, where $D_{. j}$ is complement of $A_{\cdot j}$.

\subsection{Geometric interpretation of complementary pivot method I}

It is discussed in [7] that when complementary pivot method $I$ is applied on LCP, $(q, M)$, where $M$ is a $P$-matrix of order $n$, the consecutive tableaux obtained during the algorithm represent intersections of the line $\bar{L}(n)=$ $\left\{x: x=q+z_{0} e_{n} ; z_{0}\right.$ a real valued parameter $\}$ with facets of different complementary cones in the class $\mathscr{C}(M)$. In this context, complementary pivot method I may be interpreted as a walk along a straight line cutting across different complementary cones. The sequence of cones to be crossed by this method is exactly the same as the sequence discussed in 1.2 , where the initial value of $y$ is $-\infty$. The algorithm stops when the line $\bar{L}(n)$ enters the complementary cone containing $q$.

\subsection{Notations and properties}

The following notation is used throughout the rest of the paper:

1.4.1. $\tilde{M}(n)$ denotes a $n$ by $n$ matrix whose diagonal entries are all 1 ; entries below the principal diagonal are all 2; and entries above the principal diagonal are all zero. This matrix is introduced by Murty in [7]. Notice that $\tilde{M}(n)$ is a $P$-matrix. It also is positive semidefinite, hence copositive plus. 
1.4.2. $M(n)$ denotes a $n$ by $n$ matrix such that $M(n)=\tilde{M}(n) \tilde{M}(n)^{\mathrm{T}}$. It looks like the following:

$$
M(n)=\left[\begin{array}{ccccc}
1 & 2 & 2 & \cdots & 2 \\
2 & 5 & 6 & \cdots & 6 \\
2 & 6 & 9 & \cdots & 10 \\
\vdots & \vdots & \vdots & & \vdots \\
2 & 6 & 10 & & \\
2 & 6 & 10 & \cdots & 4(n-1)+1
\end{array}\right]
$$

It is very important to notice that this matrix is symmetric and positive definite, hence also a $P$-matrix.

1.4.3. $\bar{M}(n)$ denotes a $n$ by $n$ matrix such that $\bar{M}(n)=M(n)+E(n)$; where $E(n)$ is another $n$ by $n$ matrix whose entries are all 4 . This matrix can also be verified to be a $P$-matrix.

1.4.4. $\overline{\bar{M}}(n)$ denotes a $n$ by $n$ matrix such that $\overline{\bar{M}}(n)=M(n)+F(n)$, where $F(n)$ is another $n$ by $n$ matrix whose entries are all $(-4)$, except in the first column. All entries in the first column of $F(n)$ are identically zero. $\bar{M}(n)$ can also be verified to be a $P$-matrix.

2. Computational complexity of complementary pivot method II on LCPs associated with positive definite symmetric matrices

Consider the LCP, $\left(-e_{n}, M(n)\right)$. It can be verified that $z_{1}=1, z_{i}=0 \quad(i=$ $2, \ldots, n), w_{1} \doteq 0, w_{i}=1(i=2, \ldots, n)$ is the unique solution to this problem. So $\left(z_{1}, w_{2}, \ldots, w_{n}\right)$ is the unique complementary feasible basic vector for the LCP $\left(-e_{n}, M(n)\right)$. It can also be verified that $\left(z_{1}, w_{2}, \ldots, w_{n}\right)$ is the unique complementary feasible basic vector for the LCPs, $\left(-e_{n}, \bar{M}(n)\right)$, and $\left(-e_{n}, \overline{\bar{M}}(n)\right)$.

Lemma 2.1. Consider the LCPs, $\left(-e_{n}, M(n)\right),\left(-e_{n}, \bar{M}(n)\right)$ and $\left(-e_{n}, \overline{\bar{M}}(n)\right)$. Select a complementary basic vector for these problems and let $\left(w^{*}, z^{*}\right),\left(\bar{w}^{*}, \bar{z}^{*}\right)$ and $\left(\overline{\bar{w}}^{*}, \bar{z}^{*}\right)$ be the basic solutions with respect to that same complementary basic vector in these problems. Then $\left(\bar{w}^{*}, \bar{z}^{*}\right)$ and $\left(\overline{\bar{w}}^{*}, \overline{\bar{z}}^{*}\right)$ are constant multiples of $\left(w^{*}, z^{*}\right)$.

Proof. By doing straightforward manipulations one can show that $\left(\bar{w}^{*}, \bar{z}^{*}\right)=$ $\alpha\left(w^{*}, z^{*}\right)$ and $\left(\overline{\bar{w}}^{*}, \overline{\bar{z}}^{*}\right)=\beta\left(w^{*}, z^{*}\right)$, where

$$
\alpha=\left(1+4 \sum_{i=1}^{n} z_{i}^{*}\right)^{-1} \text { and } \beta=\left(1-4 \sum_{i=2}^{n} z_{i}^{*}\right)^{-1} .
$$


Corollary 2.2. Complementary pivot method II goes through the same sequence of pivot steps when applied on either of the three LCPs $\left(-e_{n}, M(n)\right),\left(-e_{n}, \bar{M}(n)\right)$ and $\left(-e_{n}, \overline{\bar{M}}(n)\right)$.

Proof. All the three problems start with the same complementary basic vector and the same basic solution. Consequently, by the manner in which the algorithm progresses, the first pivot row would be the same ( $n$th row); resulting in the same complementary basic vector after the first pivot step in all these problems. By Lemma 2.1, the updated right hand side column vector of the two problems, $\left(-e_{n}, \bar{M}(n)\right)$, and, $\left(-e_{n}, \overline{\bar{M}}(n)\right)$ after the first pivot step would be constant multiples of the corresponding column in the updated tableau of $\left(-e_{n}, M(n)\right)$ after the first pivot. These multiplying constants must be positive since after pivoting on the $n$th row, the value of the $n$th component of the updated right hand side in each of their respective tableaux would be positive. Therefore the right hand side column vectors in all the three updated tableaux will have the same sign pattern, resulting in selection of an identical pivot row in the next pivot step. This argument can be repeated for all the consecutive steps as well. The corollary follows.

Theorem 2.3. For every $n \geq 2$, complementary pivot method II applied to $\left(-e_{n}, M(n)\right)$ goes through $2^{n}-1$ pivot steps before termination.

Proof. It can be verified that this statement is true for $n=2$. We now make the following induction hypothesis:

Induction Hypothesis: The theorem is true for the LCP of order $n-1$, $\left(-e_{n-1}, M(n-1)\right)$.

Using the induction hypothesis, we will now prove that the statement of the theorem also holds for the LCP $\left(-e_{n}, M(n)\right)$ which is of order $n$.

If the first constraint in $\left(-e_{n}, M(n)\right)$ is eliminated, as also the column vectors of $w_{1}$ and $z_{1}$, we are left with another LCP of order $n-1$, called the reduced problem which is the same as $\left(-e_{n-1}, \tilde{M}(n-1)\right)$, with the exception that its variables are called $w_{2}, \ldots, w_{n} ; z_{2}, \ldots, z_{n}$.

It should be noted that when complementary pivot method II is applied on $\left(-e_{n}, M(n)\right)$, it does not select the first row as the pivot row until it finds the unique solution of the reduced problem. By the Induction Hypothesis along with Corollary 2.2 , and since the reduced problem is nothing but $\left(-e_{n-1}, \bar{M}(n-1)\right)$, it is clear that the algorithm goes through $2^{n-1}-1$ pivot steps to find a complementary feasible basic vector for the reduced problem, before a pivot occurs in the first row. So during these $2^{n-1}-1$ pivot steps, the first row is never the pivot row, and by our knowledge about the unique complementary feasible basic vector for the reduced problem, it is clear that $\left(w_{1}, z_{2}, w_{3}, \ldots, w_{n}\right)$ is the complementary basic vector obtained after the first $2^{n-1}-1$ pivot steps. It can be easily verified that in the corresponding canonical tableau, the element of the 
updated right hand side constant in the first row is strictly negative, hence by the nature of the algorithm the next pivot row would be the first row, and $z_{1}$ replaces $w_{1}$, resulting in the canonical tableau shown in Table 1.

Table 1

An intermediate tableau, corresponding to the basic vector $\left(z_{1}, z_{2}, w_{3}, \ldots, w_{n}\right)$, when the LCP $\left(-e_{n}, M(n)\right)$, is solved by complementary pivot method II

\begin{tabular}{l|rrrrrr|rrrrrr|r}
\hline $\begin{array}{l}\text { Basic } \\
\text { variable }\end{array}$ & $w_{1}$ & $w_{2}$ & $w_{3}$ & $w_{4}$ & $\cdots$ & $w_{n}$ & $z_{1}$ & $z_{2}$ & $z_{3}$ & $z_{4}$ & $\cdots$ & $z_{n}$ & \\
\hline$z_{1}$ & -5 & 2 & 0 & 0 & $\cdots$ & 0 & 1 & 0 & -2 & -2 & $\cdots$ & -2 & \\
$z_{2}$ & 2 & -1 & 0 & 0 & $\cdots$ & 0 & 0 & 1 & 2 & 2 & $\cdots$ & 2 & 3 \\
$w_{3}$ & 2 & -2 & 1 & 0 & $\cdots$ & 0 & 0 & 0 & -1 & -2 & $\cdots$ & -2 & \\
$w_{4}$ & 2 & -2 & 0 & 1 & $\cdots$ & 0 & 0 & 0 & -2 & -5 & $\cdots$ & -6 & -1 \\
$\vdots$ & $\vdots$ & $\vdots$ & $\vdots$ & $\vdots$ & & $\vdots$ & $\vdots$ & $\vdots$ & $\vdots$ & $\vdots$ & & $\vdots$ & -1 \\
$w_{n}$ & 2 & -2 & 0 & 0 & $\cdots$ & 1 & 0 & 0 & -2 & -6 & $\cdots$ & $-(4(n-3)+1)$ & -1 \\
\hline
\end{tabular}

If the first row and columns corresponding to $w_{1}$ and $z_{1}$ are eliminated from Table 1 , we are left with a LCP of order $n-1$, called the new reduced problem, which is the same as $\left(-e_{n-1}, \overline{\bar{M}}(n-1)\right)$, with the exception that its variables are called $z_{2}, w_{3}, \ldots, w_{n} ; w_{2}, z_{3}, \ldots, z_{n}$.

Thus by Induction Hypothesis, along with Corollary 2.2, the algorithm now goes through another $2^{n-1}-1$ pivot steps to find the solution to this new reduced problem $\left(-e_{n-1}, \overline{\bar{M}}(n-1)\right)$.

Since the unique complementary feasible basic vector for the new reduced problem is $\left(w_{2}, w_{3}, \ldots, w_{n}\right)$, after all the pivot steps we will reach the complementary basic vector $\left(z_{1}, w_{2}, \ldots, w_{n}\right)$, which is the unique complementary feasible basic vector to $\left(-e_{n}, M(n)\right)$. So the algorithm terminates then.

The total number of pivot steps the algorithm goes through is therefore $\left(2^{n-1}-1\right)+1+\left(2^{n-1}-1\right)=2^{n}-1$.

Hence if the result of the theorem holds for $n-1$, it must also hold for $n$. The statement of the theorem can be easily verified for $n=2$, thus by induction it must hold for every $n \geq 2$.

\section{Computational complexity of complementary pivot method I on LCPs associated with positive definite symmetric matrices}

In order to demonstrate exponential growth of computational requirements of this algorithm, a line in $\mathbf{R}^{n}$ is constructed which cuts across all the $2^{n}$ complementary cones in the class $\mathscr{C}(M(n))$ for every $n \geq 2$. Then using this line a proper linear complementarity problem can be constructed, for which complementary pivot method I goes through $2^{n}$ pivot steps before termination.

For notational convenience, let $q(n)$ denote a column vector in $\mathbf{R}^{n}$ such that $q(n)=\left(q_{n}(n), q_{n-1}(n), \ldots, q_{1}(n)\right)^{\mathrm{T}}$. 
Lemma 3.1. If $\left(w^{*}, z^{*}\right)$ solves $\left(q(n)-\gamma^{*} e_{n}, M(n)\right)$, then there exists a real value $\lambda^{*}$ such that $\left(w^{*}, z^{*}\right)$ also solves $\left(q(n)-\lambda^{*} e_{n}, M(n)+G(n)\right)$, where $G(n)$ is a $n$ by $n$ matrix with identical rows $\left(g_{1}, \ldots, g_{n}\right)$ and $g_{1}, \ldots, g_{n}$ are arbitrary real numbers.

Proof. It can be verified that the statement of the lemma is true for $\lambda^{*}=$ $\gamma^{*}+\sum_{i=1}^{n} g_{i} z_{i}^{*}$.

Remark 3.2. Let $L(n)=\left\{x: x=q(n)-\gamma e_{n} ; \gamma\right.$ a real valued parameter $\}$ be a line in $\mathbf{R}^{n}$; and let $\mathrm{M}(n)$ be a $P$-matrix of order $n$. Then as the value of $\gamma$ varies from $-\infty$ to $+\infty$, the line $L(n)$ cuts across a sequence of complementary cones in the class $\mathscr{C}(\mathbf{M}(n))$ which is the same as the sequence of complementary cones associated with the sequence of complementary basic vectors encountered when the parametric LCP $\left(q(n)-\gamma e_{n}, \mathrm{M}(n)\right)$ is solved for the values of the parameter $\gamma$ varying from $-\infty$ to $+\infty$. This follows from the discussion in Section 1.2.

Corollary 3.3. If the line $L(n)=\left\{x: x=q(n)-\gamma e_{n} ; \gamma\right.$ a real valued parameter $\}$ cuts across all the complementary cones in the class $\mathscr{C}(M(n))$, then it also cuts across all the complementary cones in the classes $\mathscr{C}(\bar{M}(n))$ and $\mathscr{C}(\bar{M}(n))$.

Proof. We prove the statement of the corollary for the class $\mathscr{C}(\bar{M}(n))$. A similar proof can be repeated for the class $\mathscr{C}(\overline{\bar{M}}(n))$.

By Lemma 3.1, when the two parametric LCPs $\left(q(n)-\gamma e_{n}, M(n)\right)$ and $\left(q(n)-\gamma e_{n}, \bar{M}(n)\right)$ are solved for the values of $\gamma$ varying from $-\infty$ to $+\infty$, the same sequence of complementary basic vectors are encountered. Hence, by Remark 3.2, the line $L(n)$ cuts across the complementary cones associated with the same sequence of complementary basic vectors in the two classes $\mathscr{C}(M(n))$ and $\mathscr{C}(\bar{M}(n))$. The result in the corollary now follows.

Theorem 3.4. There exists a $q(n)$ such that the line $L(n)=\left\{x: x=q(n)-\gamma e_{n}\right.$; $\gamma$ a real valued parameter $\}$ cuts across all the $2^{n}$ complementary cones in the class $\mathscr{C}(M(n))$ for every $n \geq 2$.

Proof. Considering Remark 3.2, here we have to show that there exists a column vector $q(n) \in \mathbf{R}^{n}$ such that when the parametric LCP $\left(q(n)-\gamma e_{n}, M(n)\right)$ is solved for the values of $\gamma$ varying from $-\infty$ to $+\infty$, all the $2^{n}$ complementary basic vectors are encountered. We do this inductively.

It can be verified that for $n=2$ there exists such a $q(2)$ by simply letting $q(2)=(4,1)^{\mathrm{T}}$ and applying the parametric LCP algorithm, discusssed in 1.2. Now we make the following induction hypothesis:

Induction Hypothesis. There exists a $q(n-1)$ such that the parametric LCP, $\left(q(n-1)-\gamma e_{n-1}, M(n-1)\right)$, goes through $2^{n-1}-1$ pivot steps before termination. 
Using this induction hypothesis, along with Corollary 3.3 , in the rest of the proof we will show that there exists a value $\nu$ such that for every $q_{n}(n)>\nu$ and

$$
q(n)=\left(\begin{array}{l}
q_{n}(n) \\
\cdots \\
q(n-1)
\end{array}\right)
$$

the parametric LCP, $\left(q(n)-\gamma e_{n}, M(n)\right)$ goes through $2^{n}-1$ pivot steps before termination. Then by induction, the statement of the theorem must hold for every $n \geq 2$.

To show the existence of such a $\nu$, let us consider the first canonical tableau for the parametric LCP, $\left(q(n)-\gamma e_{n}, M(n)\right)$, corresponding to $\gamma=-\infty$. If the first constraint here is eliminated, as also the column vectors of $w_{1}$ and $z_{1}$, we are left with a parametric LCP of order $n-1$, called the reduced problem which is the same as the parametric LCP $\left(q(n-1)-\gamma e_{n-1}, \bar{M}(n-1)\right)$ with the exception that its variables are called $w_{2}, \ldots, w_{n} ; z_{2}, \ldots, z_{n}$. By the Induction Hypothesis and the result of Corollary 3.3, it is clear that as the value of $\gamma$ increases from $-\infty$, the reduced problem goes through $2^{n-1}-1$ pivot steps until it finds a value for $\gamma$, (say $\delta$ ), such that for every $\gamma>\delta$ the corresponding tableau for the reduced problem remains feasible. If the value of $q_{n}(n)$ is chosen large enough (say greater than $\nu$ ) the first row never becomes the pivot row during these first $2^{n-1}-1$ pivot steps.

After the first $2^{n-1}-1$ pivot steps the set of basic variables would be $\left(w_{1}, z_{2}, w_{3}, \ldots, w_{n}\right)$ and, as mentioned in Theorem 2.3, in the corresponding updated canonical tableau, the coefficients of $\gamma$ in rows 2 to $n$ would be positive, while its coefficient in the first row is negative. Thus regardless of how large the value of $q_{n}(n)$ is chosen, there exists a value $\Delta$ such that for $\gamma>\Delta$ the value of the updated right hand side (RHS) in the first row becomes negative. Hence the next pivot row would be the first row. Replacing $w_{1}$ with $z_{1}$ leads to a basic vector whose updated canonical tableau is shown in Table 2.

\section{Table 2}

An intermediate tableau corresponding to the basic vector $\left(z_{1}, z_{2}, w_{3}, \ldots, w_{n}\right)$ obtained while solving the parametric LCP, $\left(q(n)-\gamma e_{n}, M(n)\right)$ as the parameter $\gamma$ increases from $-\infty . c=2\left(q_{n}(n)-q_{n-1}(n)\right)$.

\begin{tabular}{|c|c|c|c|c|c|c|c|c|c|c|c|c|c|c|}
\hline \multirow{2}{*}{$\frac{0}{\frac{0}{0}}$} & \multirow[b]{2}{*}{$w_{\mathrm{t}}$} & \multirow[b]{2}{*}{$w_{2}$} & \multirow[b]{2}{*}{$w_{3}$} & \multirow[b]{2}{*}{$w_{4}$} & \multirow[b]{2}{*}{$\cdots$} & \multirow[b]{2}{*}{$w_{n}$} & \multirow[b]{2}{*}{$z_{1}$} & \multirow[b]{2}{*}{$z_{2}$} & \multirow[b]{2}{*}{$z_{3}$} & \multirow[b]{2}{*}{$z_{4}$} & \multirow[b]{2}{*}{$\cdots$} & \multirow[b]{2}{*}{$z_{n}$} & \multicolumn{2}{|l|}{ RHS } \\
\hline & & & & & & & & & & & & & $q(n)$ & $\gamma$ \\
\hline$z_{1}$ & -5 & 2 & 0 & 0 & $\cdots$ & 0 & 1 & 0 & -2 & -2 & $\cdots$ & -2 & $-5 q_{n}(n)+2 q_{n-1}(n)$ & 3 \\
\hline$z_{2}$ & 2 & -1 & 0 & 0 & $\cdots$ & 0 & 0 & 1 & 2 & 2 & $\cdots$ & 2 & $c+q_{n-1}(n)$ & -1 \\
\hline$w_{3}$ & 2 & -2 & 1 & 0 & $\cdots$ & 0 & 0 & 0 & -1 & -2 & $\cdots$ & -2 & $c+q_{n-2}(n)$ & -1 \\
\hline$w_{4}$ & 2 & -2 & $\begin{array}{l}0 \\
.\end{array}$ & 1 & $\cdots$ & 0 & $\begin{array}{l}0 \\
.\end{array}$ & $\begin{array}{l}0 \\
\text {. }\end{array}$ & -2 & -5 & $\cdots$ & -6 & $c+q_{n-3}(n)$ & -1 \\
\hline . & . & . & . & . & & . & . & . & $\cdot$ & . & & . & . & . \\
\hline . & . & . & - & . & & - & . & . & $\cdot$ & . & & $\cdot$ & · & $\cdot$ \\
\hline$w_{n}$ & 2 & -2 & 0 & 0 & $\cdots$ & 1 & 0 & 0 & -2 & -6 & $\cdots$ & $-(4(n-3)+1$ & $c+q_{1}$ & -1 \\
\hline
\end{tabular}


If the first row, and the columns corresponding to $w_{1}$ and $z_{1}$ are eliminated from Table 2, we are left with a parametric LCP of order $n-1$, called the new reduced problem, which is the same as the parametric LCP $\left(q(n-1)-\gamma e_{n-1}+\right.$ $\left.c e_{n-1}, \overline{\bar{M}}(n-1)\right)$, where $c=2\left(q_{n}(n)-q_{n-1}(n)\right)$. Thus, by Corollary 3.3 as the value of $\gamma$ increases, this parametric LCP goes through another $2^{n-1}-1$ pivot steps before finding a value $\theta$, such that for every $\gamma>\theta$ the final updated tableau remains feasible. Notice that by the results of Section 2, the coefficient of $\gamma$ in the first row during these last $2^{n-1}-1$ pivot steps remains positive, hence the first row does not become the pivot row in any of these last $2^{n-1}-1$ pivot steps.

The final tableau will have $\left(z_{1}, w_{2}, \ldots, w_{n}\right)$ as its complementary basic vector and it would be the complementary basic feasible vector for all values of $\gamma>\theta$.

Therefore the total number of steps this algorithm goes through for the problem of order $n$ adds up to $\left(2^{n-1}-1\right)+1+\left(2^{n-1}-1\right)=2^{n}-1$.

This result can be verified for $n=2$ as mentioned earlier. Hence, by induction, the result must hold for every $n \geq 2$.

Definition. Let $\tilde{q}(n)=q(n)-\theta e_{n}$, where $q(n)$ is a column vector in $\mathbf{R}^{n}$ and $\theta$ is a real valued constant as defined in Theorem 3.4. Notice that for every value of $\gamma$, there exist a real value $z_{0}=\theta-\gamma$ such that $\tilde{q}(n)+z_{0} e_{n}=q(n)-\gamma e_{n}$.

Theorem 3.5. Complementary pivot method I goes through $2^{n}$ pivot steps before termination when applied to the LCP $(\tilde{q}(n), M(n))$.

Proof. As discussed in 1.3, we know that the consecutive tableaux obtained in this algorithm represent intersections of the line $\bar{L}(n)=\left\{x: x=\tilde{q}(n)+z_{0} e_{n} ; z_{0}\right.$ a real value parameter $\}$ with facets of different complementary cones in the class $\mathscr{C}(M(n))$. This line is exactly the same as the line $L(n)$, defined in previous sections.

If we apply complementary pivot method I on this LCP, we observe that in the initial tableau, the value of $z_{0}$ would be $\theta-q_{1}(n)$, which corresponds to $\gamma=q_{1}(n)$. In other words this tableau represents the end of the first portion of the line $L(n)$, corresponding to $\gamma \leq q_{1}(n)$ as discussed in Theorem 3.4. As the algorithm goes on it keeps finding intersections of the line $L(n)$ with the facets of different complementary cones, cutting across these complementary cones along with $L(n)$ in exactly the same sequence as parametric LCP $\left(q(n)-y e_{n}\right.$, $M(n))$ does when the value of $\gamma$ varies from $-\infty$ to $+\infty$. We know that $\tilde{q}(n)$ lies in the complementary cone containing the last portion of $L(n)$, corresponding to $\gamma \geq \theta$. Hence this algorithm goes through $2^{n}$ pivot steps before finding the solution.

\section{Acknowledgment}

The author wishes to thank Professor Katta G. Murty for his encouragements 
and valuable comments. The author also wishes to thank the two referees for their constructive suggestions, which were helpful in improving the presentation in the paper.

Note: This work will be part of a Ph.D. dissertation to be submitted to the Department of Industrial and Operations Engineering at the University of Michigan.

\section{References}

[1] R.W. Cottle and G.B. Dantzig, "Complementary pivot theory of mathematical programming", Linear Algebra and Its Applications 1 (1968) 103-125.

[2] R.W. Cottle, "Monotone solutions of the parametric linear complementarity problem", Mathematical Programming 3 (1972) 210-224.

[3] V. Klee and G.L. Minty, "How good is the simplex algorithm", in: O. Shisha, ed., Inequalities III (Academic Press, New York, 1976).

[4] M.M. Kostreva, "Direct algorithms for complementarity problems", Dissertation, Rennselaer Polytechnic Institute (Troy, New York, 1976).

[5] C.E. Lemke, "Bimatrix equilibrium points and mathematical programming", Management Science 4 (1965) 681-689.

[6] K.G. Murty, Linear and combinatorial programming (Wiley, New York, 1976).

[7] K.G. Murty, "Computational complexity of complementary pivot methods", Mathematical Programming Study 7 (1978) 61-73.

[8] K.G. Murty, "On the number of solutions of the complementarity problems and spanning properties of complementary cones", Linear Algebra and Its Applications 5 (1972) 65-108.

[9] K.G. Murty, "On the parametric complementarity problem", Summer conference notes (The University of Michigan, 1971).

[10] K.G. Murty, "Note on a Bard-type scheme for solving the complementarity problem", Opsearch 7 (1970) 263-268.

[11] K.G. Murty, "On a characterization of P-matrices", SIAM Journal on Applied Mathematics 20 (3) (1971) 378-384.

[12] R. Saigal, "On the class of complementary cones and Lemke's algorithm", SIAM Journal on Applied Mathematics 23 (1972) 47-60.

[13] A.C. Stickney and L.T. Watson, "Digraph models of Bard-type algorithms for the linear complementarity problem", Mathematics of Operations Research 3 (1978) 322-333. 